\title{
Synthetic Plasmonic Nanocircuits and the Evolution of Their Correlated Spatial Arrangement and Resonance Spectrum
}

Yaohui Zhan ${ }^{\dagger, \ddagger}$, Lei Zhang ${ }^{\Delta, \square}$, Mohsen Rahmani ${ }^{\S}$, Vincenzo Giannini ${ }^{\Uparrow l}$, Andrey E. Miroshnichenko ${ }^{\mathfrak{L}}$, Minghui Hong", Xiaofeng $\mathrm{Li}^{\dagger, \star}$, Stefan A. Maier ${ }^{\perp, \neq}$, and Dangyuan Lei ${ }^{\#, *}$

${ }^{\dagger}$ School of Optoelectronic Science and Engineering \& Collaborative Innovation Center of Suzhou Nano Science and Technology, Soochow University, Suzhou 215006, China.

\#ey Lab of Advanced Optical Manufacturing Technologies of Jiangsu Province \& Key Lab of Modern Optical Technologies of Education Ministry of China, Soochow University, Suzhou 215006, China.

${ }^{\Delta}$ Key Laboratory for Physical Electronics and Devices, the Ministry of Education and Shaanxi Key Lab of Information Photonic Technique, School of Electronic Science and Engineering, Xi'an Jiaotong University, Xi'an 710049, China.

${ }^{\S}$ Advanced Optics and Photonics Laboratory, Department of Engineering, Nottingham Trent University, Nottingham, NG11 8NS, UK.

IInstituto de Estructura de la Materia (IEM), Consejo Superior de Investigaciones Científicas (CSIC), Serrano 121, 28006 Madrid, Spain.

${ }^{\mathrm{E}}$ School of Engineering and Information Technology, University of New South Wales, Canberra, Australian Capital Territory, Australia.

"Department of Electrical and Computer Engineering, National University of Singapore, Singapore 117576, Singapore.

${ }^{\perp}$ Chair in Hybrid Nanosystems, Nanoinstitut München, Fakultät für Physik, Ludwig MaximiliansUniversität München, 80539 München, Germany.

'Department of Physics, Imperial College London, London SW7 2AZ, UK.

\#Department of Materials Science and Engineering, City University of Hong Kong, 83 Tat Chee Avenue, Kowloon, Hong Kong, China.

${ }^{*}$ Corresponding author: Dangyuan Lei (dangylei@ cityu.edu.hk)

${ }^{\square}$ represents equal contribution 


\begin{abstract}
Optical nanocircuits, inspired by electrical nanocircuits, provide a versatile platform for tailoring and manipulating optical fields at the subwavelength scale, which is vital for developing various innovative optical nanodevices and integrated nanosystems. Plasmonic nanoparticles can be employed as promising building blocks for optical nanocircuits with unprecedentedly high integration capacity. Among various plasmonic systems, metallic aggregated nanoparticle, known as oligomers, possess great potential in constructing functional metatronic circuits. Here, the optical nanocircuits comprising special plasmonic oligomers, such as trimers with $D_{3 \mathrm{~h}}$ symmetry, quadrumers with $D_{2 \mathrm{~h}}$ symmetry, and their variants with reduced symmetry, are systematically investigated in the metatronic paradigm, both theoretically and experimentally. Our proposed circuit models, based on the displacement current in the oligomers, not only reproduce the resonance spectral details, but also retrieve many hidden physical quantities associated with their optical responses. Guided by the metatronic circuits, the spectral engineering of the oligomers with reduced geometric symmetry is predicted, and subgroup decomposition of several plasmonics quadrumers is examined. Our investigation has revealed a close correlation between the metatronic circuitry and strongly coupled plasmonic oligomers. The observed correlation of spatial arrangement and frequency response in oligomers provides a metatronic guide to modulate plasmonic responses via geometric variation.
\end{abstract}

Keywords: metal nanoparticles, plasmonic oligomers, Fano resonances, optical nanocircuits

\title{
TOC Graphic:
}

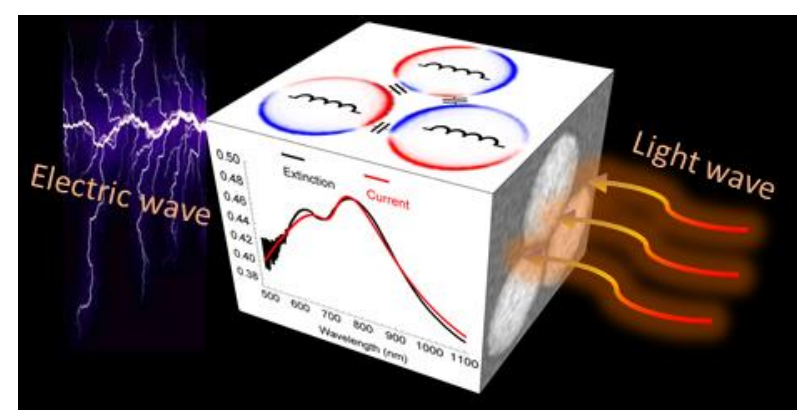


Planar aggregated plasmonic nanoparticles, so-called oligomers, have attracted considerable attention in recent years, ${ }^{1-6}$ due to their appealing applications, such as photovoltaic cells, ${ }^{7-9}$ plasmonic switches, ${ }^{10}$ color displays, ${ }^{11}$ and biological labeling techniques. ${ }^{12}$ Remarkably, plasmonic nanoparticles can concentrate light beyond the optical diffraction limit and enable truly nanoscale optical elements, optical devices, and optical circuits with unprecedented integration. In the metatronic paradigm, an individual nanoparticle can be considered as a frequency-dependent nanoelectronic element, even in the optical regime. ${ }^{13}$ A nanoparticle with negative permittivity (i.e., $\operatorname{Re}(\varepsilon)<0$ ) behaves like a nanoinductor, while that with a positive permittivity (i.e., $\operatorname{Re}(\varepsilon)>0$ ) presents as a nanocapacitor. Meanwhile, a nanoparticle can also function as a nanoresistor due to the Ohm loss resulting from the imaginary part of permittivity (i.e., $\operatorname{Im}(\varepsilon) \neq 0) .{ }^{14,15} \mathrm{~A}$ variety of metatronic circuits have been developed based on nanoparticle assemblies to explore novel optical phenomena and functionalities, ${ }^{16-18}$ and transfer specific functionalities from electronic circuits to nanophotonics. ${ }^{19-22}$ For the first time, Sun et al. experimentally realized a two-dimension optical lumped nanocircuit composed of an array of silicon nitride $\left(\mathrm{Si}_{3} \mathrm{~N}_{4}\right)$ nanorods in the mid-infrared frequency and demonstrated that the polarization of optical signals could tailor the connecting ways between nanocircuit elements. ${ }^{23}$ Later on, Caglayan et al. presented the first designable lumped nanocircuits with a tailorable response in the near-infrared frequency, making use of a simple nanorod geometry and transparent conducting oxides. ${ }^{24}$ Lately, the metatronic circuit concept has been extended to the structural dispersion of microwave waveguides, ${ }^{25,26}$ light trapping of multilayer structures $^{27}$, and plasmonic stacks based spectral filters. ${ }^{28}$ However, the nanoscale optical circuit elements have only been constructed in either one- or two-dimension regime, partially due to the tractablility of experimental demonstration.

To advance the nanoscale integration of optical circuits, Liu et al. fabricated a nanocircuit consisting of a partly loaded gold dimer, which paves the way towards the nanoparticle-based 
optical circuits. ${ }^{29}$ To assemble such nanoparticle-based optical circuits flexibly, Shi et al. invented a dynamical manipulation approach based on the atomic force microscope (AFM), through which a series of reconfigurable nanocircuits was developed successfully. ${ }^{30}$ Using the similar nanomanipulation, Park et al. assembled non-planar plasmonic oligomers on a polymethyl methacrylate (PMMA) nanohole template, providing a potential strategy for the stereoscopic nanocircuits. ${ }^{31}$

On the aspect of theoretical modeling, many endeavors were paid persistently with the focus on the nanoparticle or nanoparticle-like systems ${ }^{32-35}$. For instance, Abasahl et al. analyzed the metatronic circuits based on dolmen structures, bridging the gap between full-field electromagnetic calculations and metatronic circuits $^{33}$. Further, Benz and Attaran et al. developed theoretical circuit models to describe the coupled plasmon resonances in nanoparticle dimers, tetramers, and pentamers in both capacitive and conductive coupling regimes $^{34,36}$. Most recently, Ma et al. developed a nanophotonic circuitry consisting of nanodimers theoretically and discovered its dynamical behavior in the regimes of tristable and astable multivibrators as well as chaos generators. ${ }^{37}$

Although serveral theoretical models have been developed for the nanoparticle-based optical circuits, their associated experimental demonstrations is rarely reported. Moreover, the metatronic model of the nanoparticle-based optical circuits is also far from mature, requiring much attention to many aspects such as the robustness and consistency for different oligomers. So far, the metatronic model, that can accurately reproduce the spectral lineshape, has been limited to the relatively simple plasmonic nanostructures or metal-dielectric hybrid systems ${ }^{30,38}$. Such systems typically possess weak interactions among constituent elements. It remains challenging to capture the full details of spectral response accurately or predict the evolving plasmonic oligomers consistently due to the strong interparticle coupling that is extraordinarily complicated and extremely sensitive to the geometrical variation. ${ }^{39-44}$. An accurate circuit model is supposed to extract many hidden physical parameters that cannot be derived directly 
by full-wave simulations and promptly predict many evolved plasmonic oligomers as a supplementary of the full-wave calculation that is usually time-consuming and computationally expensive.

Here, we experimentally build a multilevel metatronic circuitry from basic elements to their composite networks to describe the spectral response of the plasmonic oligomers including the dimers, trimers, quadrumers and their variants with symmetry breaking. Unlike the existing models, we utilize a consistent circuitry wiring-way for different oligomer configurations by using which the core lumped parameters extracted from the dimer can be directly passed to the trimer and then to the quadrumers. By comparing the experimental far-field extinction spectra with the full-wave numerical simulations, the developed metatronic circuitry is verified to be robust even for complicated high-order optical nanocircuits by consistently addressing the capacitive and inductive couplings simultaneously. Based on this metatronic paradigm, the Fano resonances in these systems are successfully reproduced. Therefore, such paraigm captures the Fano-type spectral details and retrieves the rich physical information with quantitative values, including interspace capacitance, mutual coupling interaction, radiative and nonradiative resistances. By using these quantitative parameters, the metatronic circuit can be employed independently to predict the spectral evolution of a nanoparticle oligomers as its geometric symmetry is gradually broken, showing good quantitative agreement with the fullwave simulation results and experimental measurements in terms of such as the spectral contrast, spectral width, spectral decomposition, and resonance shift.

\section{- RESULTS AND DISCUSSION}

Dimer circuitry with different light polarizations. A gold $(\mathrm{Au})$ dimer is investigated first under normal illumination with two typical linear polarization directions, where an individual nanoparticle is treated as a basic element, as shown in Figure 1a. The impedance of individual nanoparticle can be represented as $Z_{p}=(-i \omega \varepsilon[\omega] \pi a)^{-1}$, where $\varepsilon$ and $a$ are the permittivity (see 
Figure S1 in Supporting Information (SI)) and the effective size of a nanoparticle, respectively; $\omega$ is the angular frequency of light. The real and imaginary parts of $Z_{p}$ originating from the complex permittivity are corresponding to the nonradiative resistance $R_{\text {nrad }}$ and the inductance $L_{p}$, respectively. The fringe capacity $\left(C_{f}=2 \pi \varepsilon_{0} a\right)$ is associated with the fringing field around the dimer configuration, and the mutual inductance $\left(L_{m}\right)$ represents the coupling strength between two nanoparticles. To further incorporate the radiative loss $\left(R_{\text {rad }}\right)$ and coupling capacity $\left(C_{p}\right)$, a more comprehensive RLC circuit is developed, as shown in Figure 1a. This circuit is employed for the full description of an individual Au nanoparticle, where the branches of $Z_{p}, R_{\text {rad }}$ and $C_{p}$ are connected parallelly in physics (see also Equation $\mathrm{S} 1$ in $\mathrm{SI}$ ).

For the circuitry consisted of dimers, the individual nanoparticle is treated to be connected in series or in parallel with the other one, depending on the polarization of incident light. As can be seen in Figure 1b, when the electric field vector is perpendicular to the dimer axis (in this case, the polarization angle is defined as $90^{\circ}$ for convenience), the metallic particles can be deemed as a shunt-connected nanocircuit (see also Figure $\mathbf{S} 2$ in SI), and the $L_{m}$ is negligible due to the weak coupling between two nanoparticles. On the other hand, when the electric field vector is parallel to the dimer axis (see Figure 1c), the dimer components can be regarded as connecting in series along the electric field (see also Figure S3 in SI). 


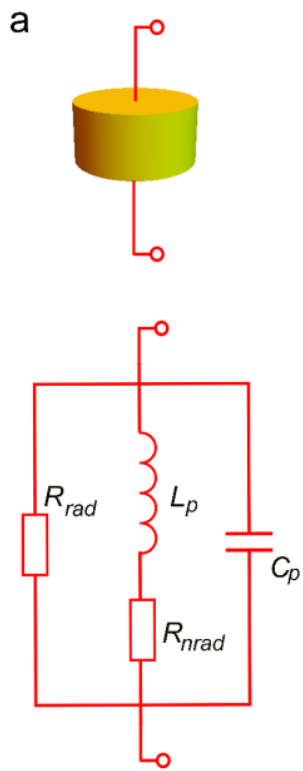

b

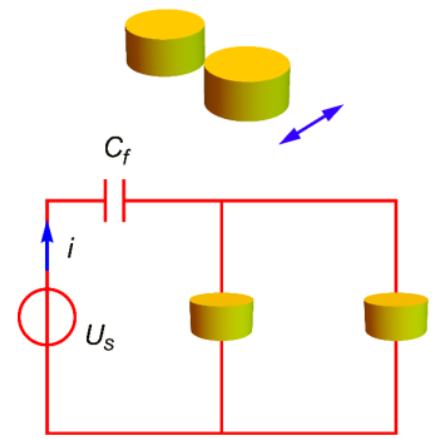

d

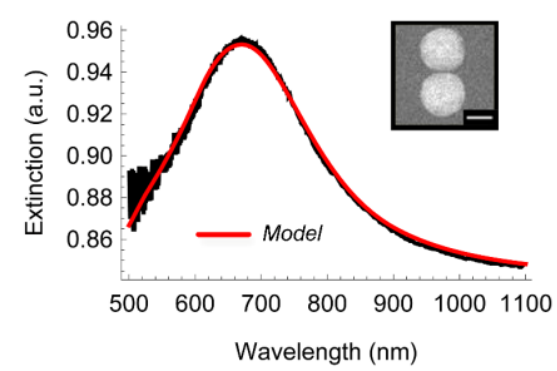

C

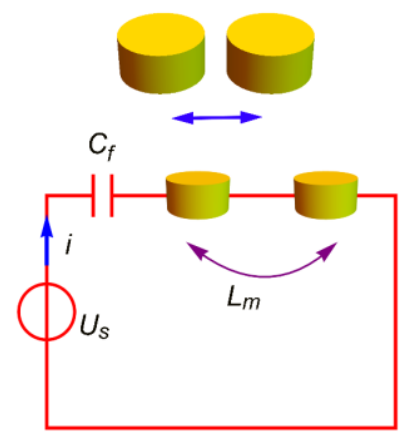

e

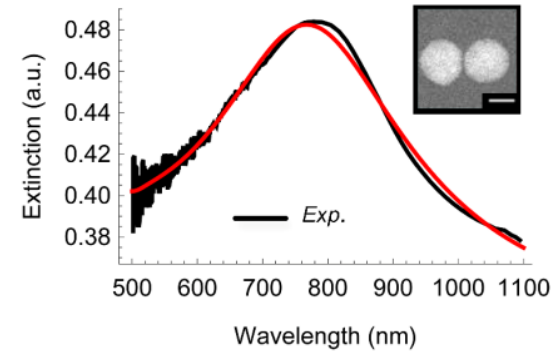

Figure 1. Schematic of metatronic nanocircuits and their polarization-dependent responses. (a) Metatronic description of an individual plasmonic particle. (b) Metatronic description of a shunt-circuit constituted by a plasmonic dimer under the illumination of electric field (blue arrow) perpendicular to the dimer axis. (c) Metatronic description of a series circuit formed by a plasmonic dimer under the illumination of electric field parallel (blue arrow) to the dimer axis. The constant voltage source $\left(U_{s}\right)$, the fringe capacitance $\left(C_{f}\right)$ and the mutual induction coefficient $\left(L_{m}\right)$ are related to the light stimulation, the ambient capacitive reactance, and the interparticle coupling, respectively. (d), (e) The measured (black line) extinction spectra and the modeled polarization currents (red line) for two polarized configurations corresponding to (b) and (c), respectively. Insets: SEM images of the fabricated dimers; the scale bar is $100 \mathrm{~nm}$.

As shown in Figure 1d and e, the polarization current in the circuit model (denoted by the blue single-headed arrows in Figure $\mathbf{1 b}$ and c) matches well with the experimental extinction spectra, capturing the detailed features of the measured spectra, such as the spectra shape, resonance frequency, and quality factor. The key parameters for the polarization-dependent dimer circuits are listed in Table 1 . As the polarization angle equals to $0^{\circ}, L_{\mathrm{m}} \approx 28.39 \mathrm{fH}, \mathrm{C}_{f} \approx$ 83.66 $a \mathrm{~F}$, and $R_{\text {rad }} \approx 169.17 \Omega$. The effective size of nanoparticle is adjusted to be $a=103 \mathrm{~nm}$ for precisely reproducing the extinction spectra, which agrees well with the size observed in scanning electron microscopy (SEM) images. As the polarization angle changes to be $90^{\circ}$, the value of $R_{\text {rad }}$ reduces significantly and results in an increased quality factor, which corresponds 
to the weakened radiative damping. However, the values of $C_{p}$ and $C_{f}$ change little, indicating the capacitance of the nanoparticles and the fringe field around the dimers are almost unaffected as the external field switches. These invariants of the nanoparticle allow us to treat the circuit model of each nanoparticle as a relatively independent system and only focus on the variables arising from the interaction with the surrounding. Therefore, the parameters for the individual nanoparticle ( $\left.a=103 \mathrm{~nm}, C_{p}=3.85 a \mathrm{~F}\right)$ and the fringe capacity $C_{f}$ remain unchanged throughout the investigation. Therefore, one can solve extremely challenging high-order problems. It should be noted that the order of our circuit model is proportional to the number of plasmonic particles, because each additional particle will bring forth an additional capacitive or inductive branch to the circuit. Therefore, the dimers, trimers, and quadrumers can be considered as second-, third-, and fourth-order nanocircuits, respectively.

Table 1. Key parameters for the metatronic nanocircuits of the dimer configuration under two linearly polarized illuminations

\begin{tabular}{ccccc}
\hline & $C_{p}(a \mathrm{~F})$ & $L_{m}(f \mathrm{H})$ & $C_{f}(a \mathrm{~F})$ & $R_{\text {rad }}(\Omega)$ \\
\hline$\|\left[0^{\circ}\right]$ & 3.85 & 28.39 & 83.66 & 169.17 \\
$\perp\left[90^{\circ}\right]$ & 3.85 & N.A. & 83.60 & 12.69 \\
\hline
\end{tabular}

Trimer circuitry and its tunability on geometric evolutions. A plasmonic trimer is built to promote the flexibility of the optical circuit system. As shown in Figure 2a, the plasmonic trimer consists of three identical nanoparticles with equal interspace between every two nanoparticles. The interparticle capacities between each pair of particles are denoted as $C_{a b}, C_{b c}$, and $C_{a c}$, respectively. The extinction spectra are independent of the polarization orientation in the case of normal illumination due to the $\mathrm{C}_{3}$ symmetry. Without loss of generality, the electric field polarization is chosen to be parallel to the centerline of A and C. As shown in Figure 2b, 
the nanocircuit is developed (see also Figure S4 in SI for details) by handling both the capacitive and inductive couplings in the system properly. The logically consistent circuit can be intuitively described as follows: firstly, the nanocapacitor is inserted between every two nanoparticles, representing the interparticle capacitance coupling; then all the nanoelements are connected sequentially along the direction of displacement current; finally, the mutual inductance is labeled between nanoparticles that are precisely parallel to the electric field for representing the interparticle inductive coupling (see Figure S2-S5 for details). Similar to the dimer, the mutual inductance in the trimer is also taken into account for the nanoparticles parallel to electric field (i.e., A and C). As demonstrated in Figure 2c, a pronounced Fano lineshape appears in the extinction spectrum, which matches well with the modeling current in the nanocircuit. This Fano lineshape is due to the near-field coupling between the superradiant mode and subradiant mode. The detailed parameters of the trimer nanocircuit are listed in Table 2. The interparticle capacities of $C_{a b}$ and $C_{b c}$ are equal to each other due to the mirror symmetry. In contrast, $C_{a b}$ (or $\left.C_{b c}=175 a \mathrm{~F}\right)$ is greater than $C_{a c}(108 a \mathrm{~F})$, which is ascribed to the smaller projection distance of the interspace $\mathrm{AB}$ (or BC) onto the direction of electric field, as compared to AC.

From an intuitive perspective, the trimer configuration can also be regarded as a dimer (e.g., AC) with an introduced third nanoparticle $(e . g ., \mathrm{B})$. It is found that the mutual inductance $L_{m}$ is almost unchanged, which indicates that the third nanoparticle has a negligible influence on the coupling strength within the dimer. Therefore, the superradiant mode of dimer configuration is preserved in the trimer configuration. As a result, the dimer and the trimer configurations share a similar dipolar extinction peak at $\sim 780 \mathrm{~nm}$, as shown in Figure 1c and $\mathbf{2 d}$. Different from the invariance of the coupling strength $\left(L_{m}\right)$, the interparticle capacitances $\left(C_{a b}, C_{a c}\right.$, and $\left.C_{b c}\right)$ and the radiation resistance $\left(R_{\text {rad }}\right)$ of the trimers change considerably. As listed in Table 2, the radiation resistances of the nanoparticle $\mathrm{B}\left(R_{\text {radl }}\right)$ and $\mathrm{A}(\mathrm{C})\left(R_{\text {rad } 2}\right)$ turn into $\sim 34 \Omega$ and $\sim 50 \Omega$ from $\sim 169 \Omega$, respectively. The reduction of $R_{\text {rad }}$ suggests an increased spectral quality factor. 
As shown in Figure 2c, the spectral widths of two extinction peaks are much narrower than the counterpart of the bright mode of parallel-aligned dimers, which is consistent with prediction. By comparing with Figure 1d and e, it can be concluded that the blue- and red-side bright modes are dominantly contributed from the subgroups of $\mathrm{B}$ and $\mathrm{AC}$, respectively.

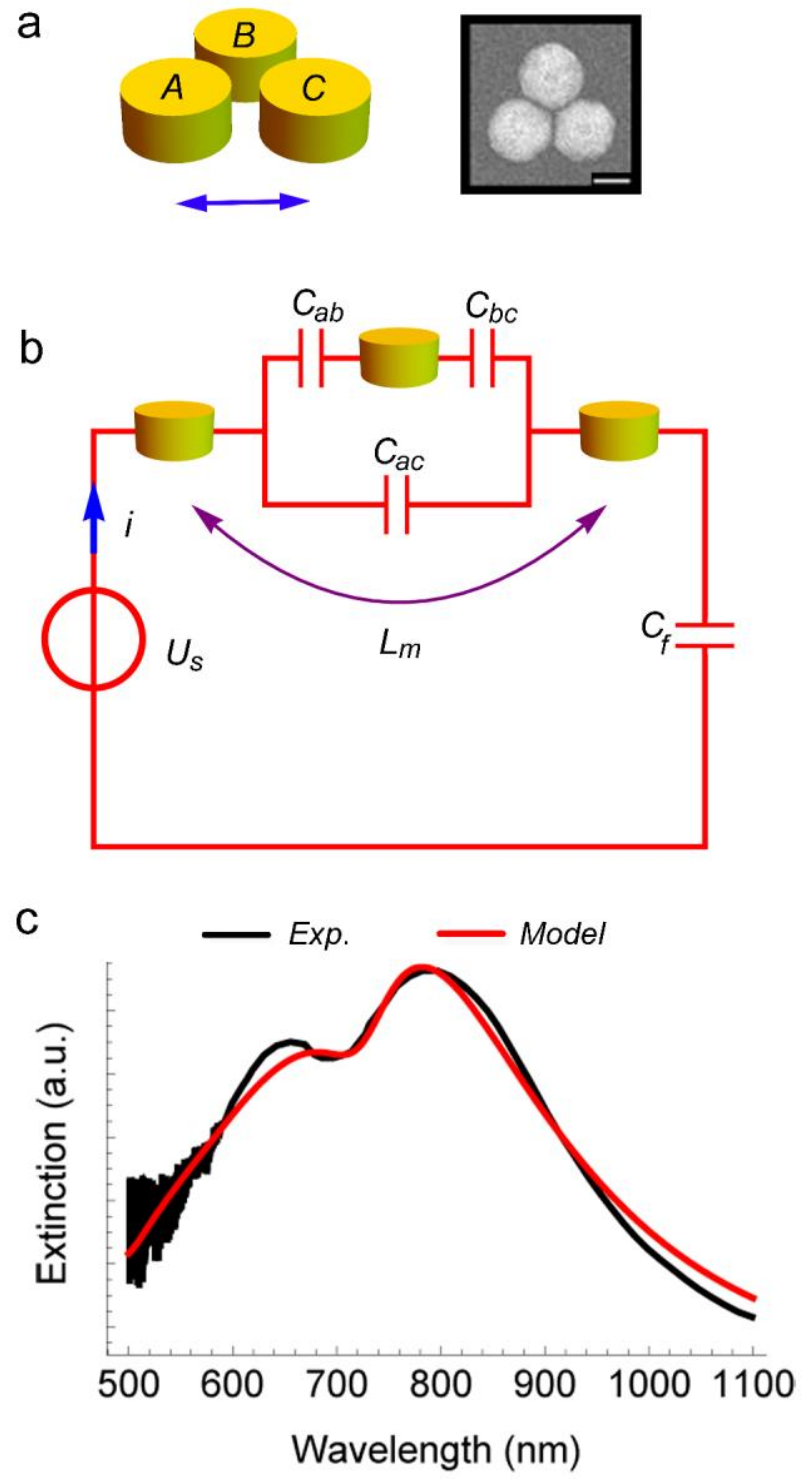

Figure 2. Optical nanocircuit built by a plasmonic trimer with $C_{3}$ symmetry. (a) Trimer configuration with equivalent interspaces and particle sizes. The labels (A, B, and C) are utilized to distinguish the relative location of each nanoelement. SEM image of the fabricated trimers with a scale bar of $100 \mathrm{~nm}$. (b) Electrical circuit model for the plasmonic trimer; (c) Comparison of experimental extinction spectra (black line) and modeling polarization current (red line) for the trimer in (a). 
Table 2. Key parameters for the metatronic nanocircuits of the trimer configuration

\begin{tabular}{ccccccc}
\hline Parameter & $\mathrm{C}_{\mathrm{ab}}(a \mathrm{~F})$ & $\mathrm{C}_{\mathrm{bc}}(a \mathrm{~F})$ & $\mathrm{C}_{\mathrm{ac}}(a \mathrm{~F})$ & $\mathrm{L}_{\mathrm{m}}(f \mathrm{H})$ & $\mathrm{R}_{\mathrm{rad} 1}(\Omega)$ & $\mathrm{R}_{\mathrm{rad} 2}(\Omega)$ \\
\hline Value & 175 & 175 & 108 & 28.40 & 34.43 & 50.20 \\
\hline
\end{tabular}

In contrast to plasmonics dimers, plasmonic trimers sustain an additional degree of freedom for engineering their spectral responses and near-field profiles by breaking the $\mathrm{D}_{3 \mathrm{~h}}$ symmetry. As shown in Figure 3a, when the trimer configuration holds a $\mathrm{C}_{3}$ symmetry, the extinction spectrum is independent of the polarization of the incident light. However, when the relative position of particle B is shifted away from the centerline of particles A and C gradually, in particular, $\angle \mathrm{ACB} \cong 90^{\circ}$ and $120^{\circ}$, the resulted symmetry breaking alters its optical response as shown in Figure 3a. A larger angle of $\angle A C B$ will result in a reduction in the spectral contrast of the extinction spectra, which is almost disappeared at $\angle \mathrm{ACB} \cong 120^{\circ}$. Numerical simulations further confirm the experimental results. From the viewpoint of the metatronic circuitry, $C_{a c}$ remains almost unchanged during the geometrical evolution; however, $C_{a b}$ or the ratio of $C_{a b}$ to $C_{a c}\left(\right.$ i.e., $\left.C_{a b} / C_{a c}\right)$ decreases gradually. As shown in Figure 3b, the spectra contrast is surely dependent on the ratio $C_{\mathrm{ab}} / C_{a c}$. Specifically, when $C_{a b} / C_{a c}$ becomes ultra-small, the Fano dip even disappears completely, which indicates that the trimer degenerates into two linearly coupled dimers. It is noted that, for predicting the spectral response of the trimers with reduced symmetry, all the circuitry parameters are the same as that with $\mathrm{D}_{2 \mathrm{~h}}$ symmetry, except $C_{\mathrm{ab}}$ and $C_{a c}$.

To explore the microscopic origin of the evolutionary trimers, normalized field profiles and charge densities at typical spectral positions are calculated numerically. The spectral response of trimer configuration with $\mathrm{C}_{3}$ symmetry in Figure $2 \mathrm{c}$ is the same as those indicated in Figure 3a due to the polarization-independence. As shown in Figure 3c, at the peak frequencies, the electric field is mainly localized at the interspace of the nanoparticle pair in parallel to the electric vector, and the coupling strength between them is almost unchanged during the 
evolution (e.g., $\mathrm{A}$ and $\mathrm{C}$ in $\mathrm{C} 12, \mathrm{C} 32$, and $\mathrm{C} 42$ ). When the centerline of the nanoparticle pair forms an acute angle $\left(<90^{\circ}\right)$ to the electric vector, the coupling strength is dramatically reduced,
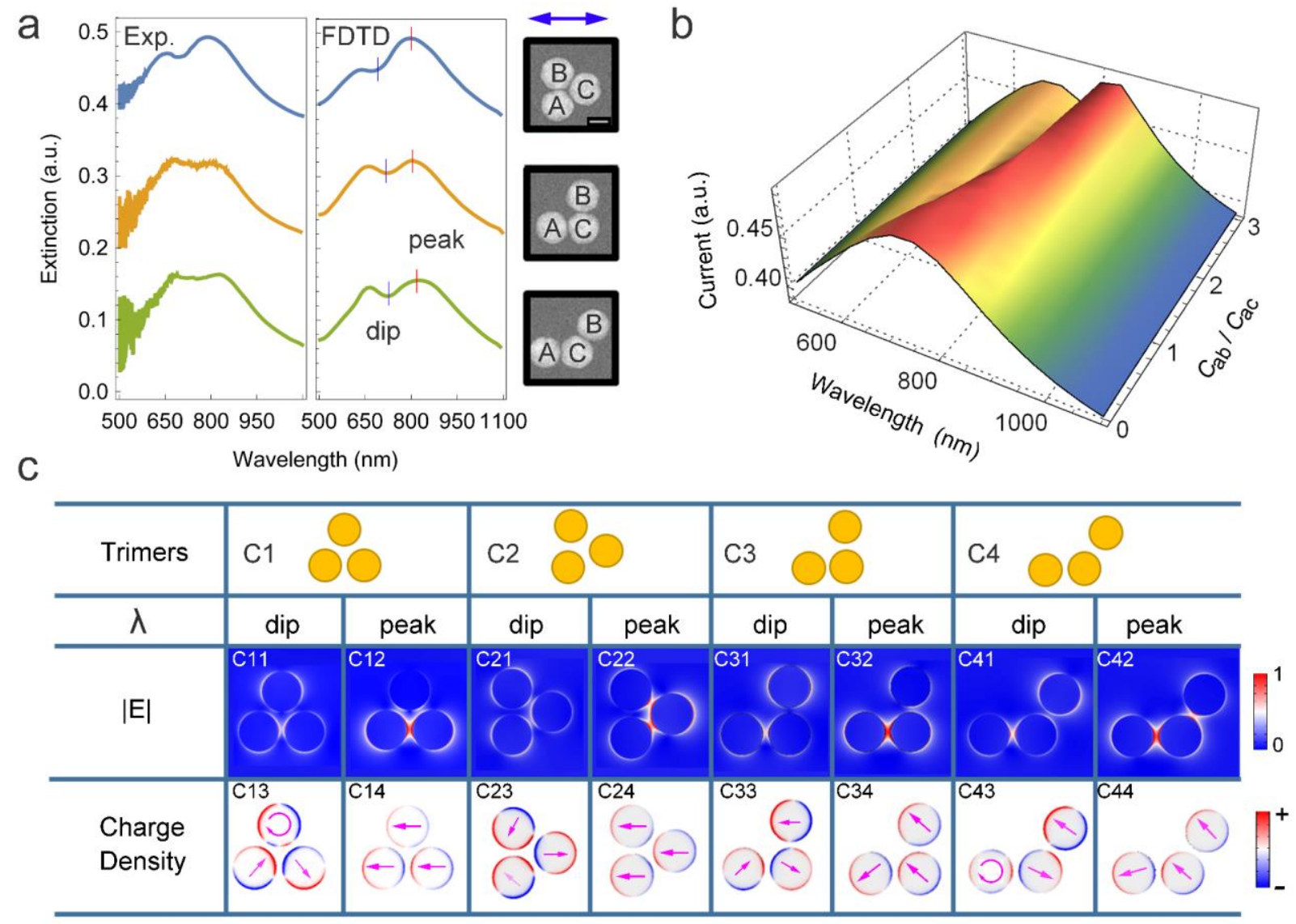

Figure 3. Dependence of the resonant responses of the plasmonic trimers with symmetry breaking. (a) Comparison of measured (left panel) and calculated (middle panel) extinction spectra of the trimers with $\mathrm{D}_{3 \mathrm{~h}}$ symmetry and its $\mathrm{C}_{2 \mathrm{v}}$ variants; SEM images (right panel) for the evolutive trimers with a scalebar of $100 \mathrm{~nm}$. (b) Polarization current as a function of the ratio $C_{a b} / C_{a c}$ and the wavelength. (c) Normalized electric field and charge density distributions in various trimers (i.e., $\mathrm{C} 1-\mathrm{C} 4$ ) at characteristic frequencies as labeled in (a). The dipole moments are denoted as pink arrows for clearly showing the mode state.

which is roughly in proportion to the intersection angle (e.g., B and $\mathrm{C}$ in $\mathrm{C} 22$ and $\mathrm{C} 42$ ). Specifically, as the intersection angle is equal to $90^{\circ}$, the coupling strength diminishes completely (e.g., $\mathrm{B}$ and $\mathrm{C}$ in $\mathrm{C} 32$ ). The field distribution phenomenally confirms the reasonability of the assumptions that the mutual inductance is only considered for the nanoparticle pair of $\mathrm{AC}$ and its magnitude remains the same throughout the circuital evolution. The extinction intensity is determined by the total dipole moment of the plasmonic system, 
which are labeled schematically on the distributions of charge density in Figure 3c. Initially, the dipole moment of individual particles at the red-side peak frequency is well-aligned to each other, like C14 and C24 as shown in Figure 3c. However, as the trimer configuration evolves, the direction of the dipole moment in each nanoparticle gradually becomes misaligned (i.e., C34 and C44), which inevitably leads to a reduction of the total dipole moment and then a decrease in the scattering intensity of the bright mode. In contrast, along with the symmetry breaking, the total dipole moment of the dark mode gradually increases (as shown in C13, C23, C33, and C43), making the dark mode become not so "dark". Because of the two reasons above, the spectral contrast of extinction spectra decreases gradually with the symmetry breaking.

Quadrumer circuitry with $\mathbf{D}_{2 \mathrm{~h}}, \mathbf{C}_{2 \mathrm{v}}$, and reduced symmetries. The metatronic model is further adopted to treat the optical response of more complex oligomers. Based on our previous work, the Fano lineshape of plasmonic clusters could be decomposed into several spatial subgroups. ${ }^{45}$ For instance, as shown in Figure 4a, the quadrumer ABCD can be decomposed into two subgroups of $\mathrm{BC}$ and $\mathrm{AD}$. Interestingly, as shown in Figure 4c, the measured extinction spectra of $\mathrm{BC}$ (blue line) and $\mathrm{AD}$ (red line) overlap well with the blue-side and the red-side extinction peaks of $\mathrm{ABCD}$, respectively. To explain the experimental observation from a metatronic perspective, the optical circuitry for the quadrumers (see also Figure S5 in SI for details) is developed as shown in Figure 4b. The key parameters for the nanocircuit of the quadrumer are listed in Table 3. In general, the modeling procedure of the quadrumer nanocircuit is logically consistent to that of the trimers, i.e., firstly labeling the nanocapacitor $\mathrm{C}_{\mathrm{ij}}(\mathrm{i}, \mathrm{j}=\mathrm{a}, \mathrm{b}, \mathrm{c}, \mathrm{d})$ between the nanoparticle elements of $\mathrm{I}$ and $\mathrm{J}(\mathrm{I}, \mathrm{J}=\mathrm{A}, \mathrm{B}, \mathrm{C}, \mathrm{D})$, then connecting the $\mathrm{B}, \mathrm{C}$, and $\mathrm{C}_{\mathrm{ad}}$ branches parallelly, and finally connecting the shunt part with $\mathrm{A}$ and $\mathrm{D}$ in series. It is worth noting that constructing such a metatronic circuit must follow the rule, i.e., the actual loop of displacement current in the system; otherwise, the spectral reproduction of such plasmonic oligomers is nearly impossible as the circuit order is $\geqslant 3$. As 
shown in Figure 4e, the flow of displacement current (indicated by red arrows) is completely consistent with the model. As a consequence, the displacement current in the metatronic circuit reproduces the extinction spectra successfully in the optical domain, as shown in Figure $4 \mathrm{~d}$. Similar to the optical subgroup decomposition, the quadrumer circuit can also be divided into $\mathrm{AD}$ and $\mathrm{BC}$ branches. As the branch of $\mathrm{BC}$ is short-circuited (i.e., only the branch of $\mathrm{AD}$ is left), the quadrumer nanocircuit degenerates exactly into the dimer model as discussed in Figure 1b. On the other hand, if the AD branch is short-circuited, the nanocircuit becomes similar to the dimer model as discussed in Figure 1c. To further unveil the physical picture, the normalized displacement field $(|D|)$ and the flow of displacement current are given in Figure 4e-h. The black circle $(\bullet)$ and square ( $\bullet$ denote the blue-side and red-side resonance frequencies of the quadrumer $\mathrm{ABCD}$, respectively; the blue circle $(\bullet)$ and red square ($\square)$ represent the resonance frequencies of the dimers $\mathrm{BD}$ and $\mathrm{AD}$, respectively. In comparing Figure 4e with Figure 4g, the displacement field in the subgroup BC is much stronger than the counterpart in the subgroup $\mathrm{AD}$, and is comparable to that of the dimer $\mathrm{BC}$ alone, which indicates the peak at $\sim 670 \mathrm{~nm}$ of the quadrumer $\mathrm{ABCD}$ originates from the subgroup $\mathrm{BC}$. Similar behaviors can also be found for the dimers and the subgroup AD in comparing Figure 4f with Figure 4h. 
a

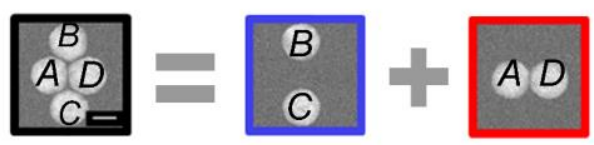

b

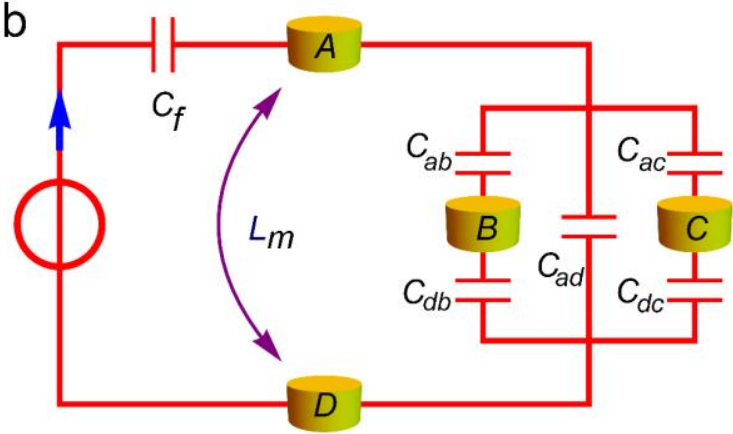

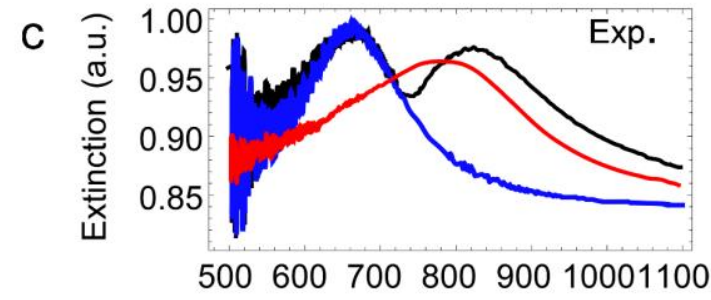

d

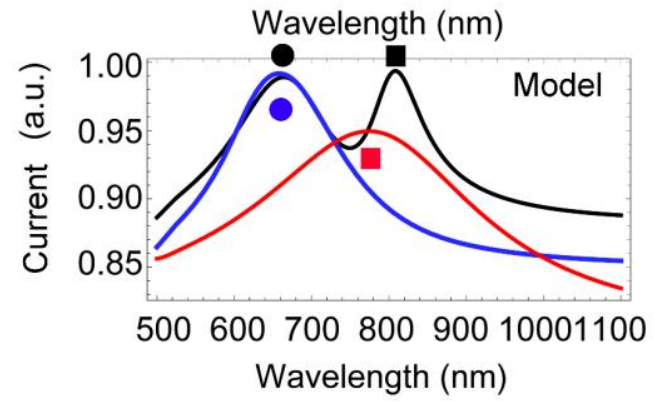

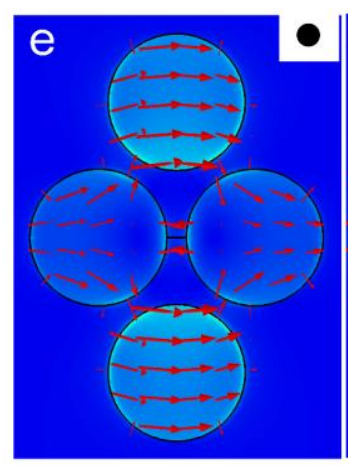
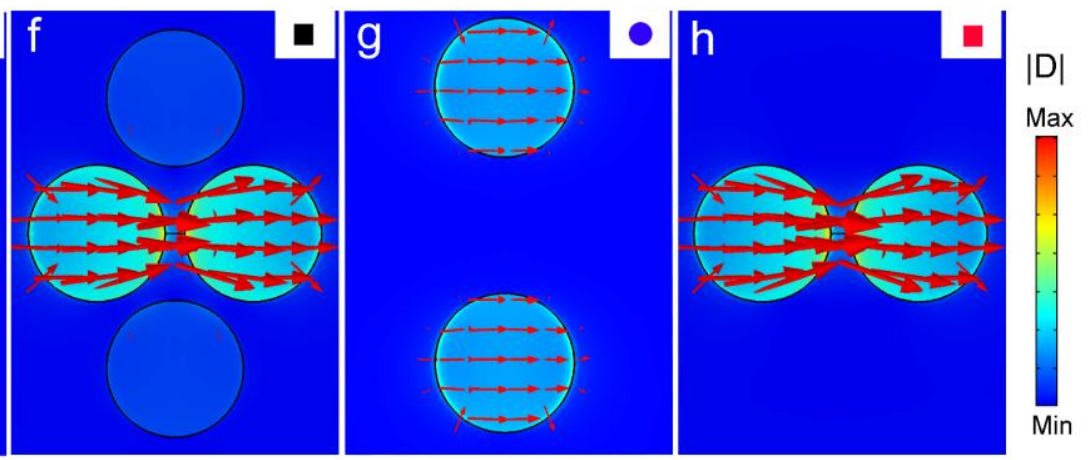

Figure 4. Optical nanocircuit built by a plasmonic quadrumer with $D_{2 \mathrm{~h}}$ symmetry. (a) The schematic diagram for subgrouping the quadrumer $\mathrm{ABCD}$ into two dimers of $\mathrm{BC}$ and $\mathrm{AD}$; the separated dimers are fabricated with the same size and same spatial location as the corresponding parts in the quadrumers. (b) The metatronic circuit of quadrumers, where B and C are shunt-connected firstly and then series-connected to A and D. (c) The measured extinction spectra and (d) the modeling electrical currents for the quadrumer (black line) and the dimer subgroups (blue and red lines). The normalized electric displacement fields of the quadrumer (e, f) and the dimer subgroups $(\mathrm{g}, \mathrm{h})$ at characteristic frequencies labeled in (d). The red arrows are the normalized displacement current on a logarithmic scale.

Table 3. Key parameters for the metatronic nanocircuits of the quadrumer configurations

\begin{tabular}{cccccc}
\hline Parameter & $\mathrm{C}_{\mathrm{ab}}(a \mathrm{~F})$ & $\mathrm{C}_{\mathrm{ad}}(a \mathrm{~F})$ & $\mathrm{L}_{\mathrm{m}}(f \mathrm{H})$ & $\mathrm{R}_{\mathrm{rad} 1}(\Omega)$ & $\mathrm{R}_{\mathrm{rad} 2}(\Omega)$ \\
\hline Value & 175 & 274 & 6.39 & 39.09 & 80.54 \\
\hline
\end{tabular}

Finally, the quadrumer with $\mathrm{C}_{2 \mathrm{v}}$ symmetry and its variants are also investigated using the metatronic circuit, which further demonstrates the convenience of circuit models in predicting the tricky optical response. As shown in the rightmost column of Figure 5, the quadrumer I 
with $\mathrm{C}_{2 \mathrm{v}}$ symmetry has only one mirror-symmetyr plane, which results in a particular capacitance distribution, i.e., $C_{a b}\left(C_{d b}\right)=C_{a c}\left(C_{d c}\right)$ and $C_{a b}\left(C_{a c}\right) \neq C_{d b}\left(C_{d c}\right)$. Although quadrumer I and its variants of II and III have a reduced symmetry compared to that of the $\mathrm{D}_{2 \mathrm{~h}}$ quadrumer, their circuit structure has no fundamental difference from that of Figure $4 \mathrm{~b}$, so there is no need to rebuild the circuit architecture, except to modify the specific assignment of nanocapacitors. In this respect, the advantages of the metatronic circuit are well represented. It is not only fast in computation but also has strong adaptability, i.e., no need to remodel every structural change like the full-wave simulations. The quadrumer evolution from type I to II and then III can be treated as a rotation of the nanoparticle B anticlockwise around A. The response to the light excitation predicted by the circuitry is displayed in Figure 5a, which are then verified by the experiment and simulations. As observed in Figure 5, the metatronic circuit correctly predicts the spectral contrast of the extinction spectra for these special quadrumers, which is characterized by the higher blue-side peak relative to the red-side one. Furthermore, the tendency of the spectral contrast is also captured by the circuit model. As shown in Figure $5 \mathrm{a}$, in the process of evolution, $C_{b d}$ is reduced gradually due to enlarged interspace, and the spectral contrast between the blue-side and red-side peaks is reduced concomitantly. The decrease of spectral contrast is mainly caused by the increase of the red-side peak, which arises from the decrease of the capacitance in the B branch and leads to an increase of the current in the AD branch based on Kirchhoff's laws.
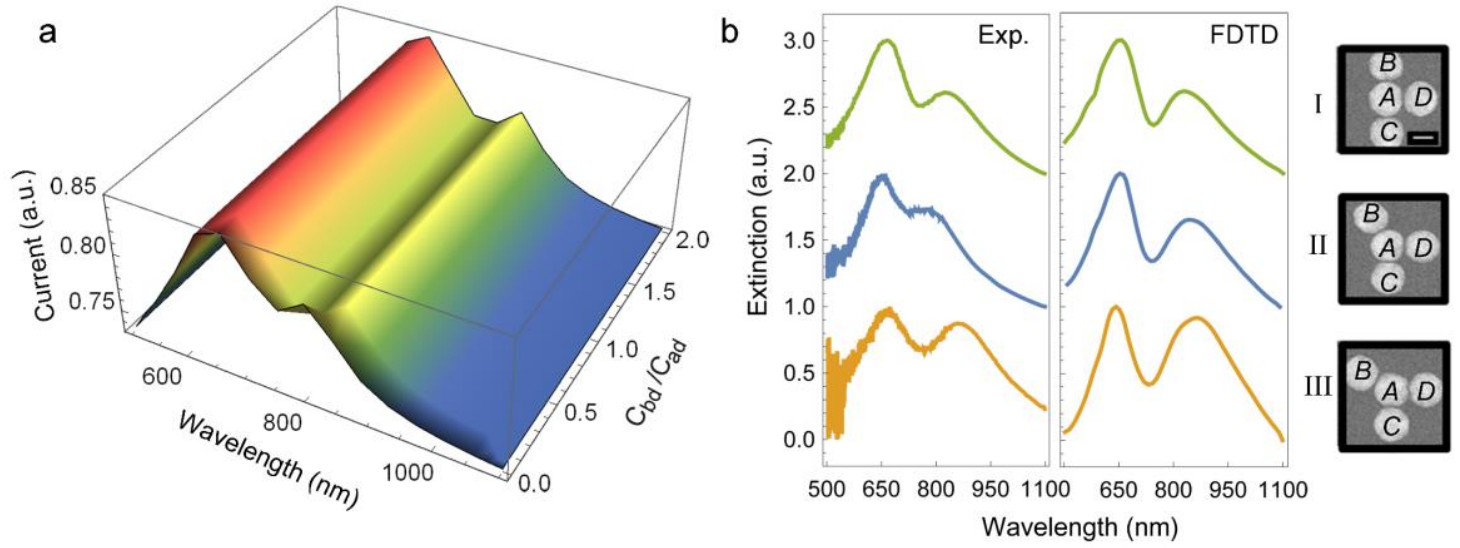
Figure 5. (a) Evolution of polarization current given by the metatronic circuit model of the quadrumer with $\mathrm{C}_{2 \mathrm{v}}$ symmetry and its variants. (b) The measured and calculated extinction spectra for the corresponding quadrumers listed in the right-most column. The scalebar of SEM images is $100 \mathrm{~nm}$.

\section{- CONCLUSION}

In summary, we comprehensively investigate the optical response of plasmonic oligomers to light excitation from the metatronic perspective. After retrieving the elementary electrical parameters of the nanocircuit from a dimer configuration under polarized optical excitations, high-order nanocircuits, based on trimers, quadrumers, and their variants with reduced symmetry, can then be built theoretically, which well explain the experimental extinction spectra. The self-consistent metatronic model captures the full spectral features of the complicated Fano resonances, shines more light on spatial subgroup decomposition, and predicts well the spectral evolution with structural changes. In particular, benefiting from addressing the microscopic inductive and capacitive couplings properly, the metatronic circuitry can achieve one to one correspondence to the complex plasmonic structures in space, and thus provides a rational way to engineering the spectral details via geometrical evolution.

\section{METHODS}

Sample preparation and optical measurements: The Au oligomers with different shapes were fabricated using electron beam lithography. A 3-nm-thick titanium (Ti) film was deposited on the substrate by e-beam evaporation to increase the adhesion between Au and quartz substrate. A 60 -nm-thick Au film was then evaporated on the substrate followed by a spin-coating of 50nm-thick hydrogen silsesquioxane (HSQ) film as a negative electro-resist. After baking the sample at $200{ }^{\circ} \mathrm{C}$ for $2 \mathrm{~min}$, a combined process of e-beam exposure, chemical development, and iron milling was performed to create well-defined Au nanodisk oligomers on the substrate. The developed morphology was characterized by high-resolution SEM. Optical transmission 
spectra were measured using Fourier transform infrared spectroscopy (FTIR, Bruker Hyperion 2000), and the extinction, defined as (1 - Transmission), was then calculated.

Full-wave numerical simulations and metatronic modeling: The 3D optical calculation was carried out using a commercially available finite-difference-time-domain code (Lumerical FDTD). The permittivity of the Au material was obtained from the experimental data ${ }^{46}$. From the electric field distribution, we calculate the charge density distribution by applying Gauss' law, $\rho=\varepsilon_{0} \nabla \cdot E$, where $E$ is the electric field vector in the near field, $\rho$ the charge density, and $\varepsilon_{0}$ the permittivity in vacuum. The metatronic circuitry is developed in a rational way based on the microscopic information (such as the direction, magnitude, and spatial distribution) of the display current and the charge density. All the circuitry is frequency-dependent, and the detail of the circuitry modeling is attached in the supporting file.

\section{- ASSOCIATED CONTENT}

\section{(1)Supporting Information}

This material is available free of charge via the internet at http://pubs.acs.org.

Frequency-dependent dielectric constant used in the circuit models, full circuitry of dimers, full circuitry of trimers, and full circuitry of quadrumers, detailed electrical parameters for the metatronic nanocircuits of the trimer and quadrumer configurations.

\section{Notes}

The authors declare no competing financial interest.

\section{- ACKNOWLEDGMENTS}

Y. Zhan thanks the support by the Natural Science Foundation of Jiangsu Province (Grant No. BK20181167), the National Natural Science Foundation of China (Grant No. 61405132), and the Opening Project of State Key Laboratory of High Performance Ceramics and Superfine Microstructure (Grant No. SKL201912SIC). L. Zhang thanks the Fundamental Research Funds 
for the Central Universities of Xi' an Jiaotong University (Grant No. Z201805196) and Natural Science Foundation of Shaanxi Province (Grant No. 2018JM6001). M. Rahmani acknowledges support from the Royal Society and the Wolfson Foundation. D.Y. Lei acknowledges the financial support by the Research Grants Council of Hong Kong (GRF Grant No. 15303417).

\section{- REFERENCES}

(1) Sheldon, M. T.; Groep, J. van de; Brown, A. M.; Polman, A.; Atwater, H. A. Plasmoelectric Potentials in Metal Nanostructures. Science 2014, 346 (6211), 828-831. https://doi.org/10.1126/science.1258405.

(2) Fan, J. A.; Wu, C.; Bao, K.; Bao, J.; Bardhan, R.; Halas, N. J.; Manoharan, V. N.; Nordlander, P.; Shvets, G.; Capasso, F. Self-Assembled Plasmonic Nanoparticle Clusters. Science 2010, 328 (5982), 1135-1138. https://doi.org/10.1126/science.1187949.

(3) Hentschel, M.; Saliba, M.; Vogelgesang, R.; Giessen, H.; Alivisatos, A. P.; Liu, N. Transition from Isolated to Collective Modes in Plasmonic Oligomers. Nano Lett. 2010, 10 (7), 2721-2726. https://doi.org/10.1021/nl101938p.

(4) Navarrete, J.; Siefe, C.; Alcantar, S.; Belt, M.; Stucky, G. D.; Moskovits, M. Merely Measuring the UV-Visible Spectrum of Gold Nanoparticles Can Change Their Charge State. Nano Lett. 2018, 18 (2), 669-674. https://doi.org/10.1021/acs.nanolett.7b02592.

(5) Haran, G.; Chuntonov, L. Artificial Plasmonic Molecules and Their Interaction with Real Molecules. Chem. Rev. 2018, $118 \quad$ (11), 5539-5580. https://doi.org/10.1021/acs.chemrev.7b00647.

(6) Zhan, C.; Liu, B.-W.; Huang, Y.-F.; Hu, S.; Ren, B.; Moskovits, M.; Tian, Z.-Q. Disentangling Charge Carrier from Photothermal Effects in Plasmonic Metal Nanostructures. Nat. Commun. 2019, 10 (1), 2671. https://doi.org/10.1038/s41467-01910771-3.

(7) Amiri, O.; Salavati-Niasari, M.; Mir, N.; Beshkar, F.; Saadat, M.; Ansari, F. Plasmonic Enhancement of Dye-Sensitized Solar Cells by Using Au-Decorated Ag Dendrites as a Morphology-Engineered. Renewable Energy 2018, 125, 590-598. https://doi.org/10.1016/j.renene.2018.03.003.

(8) Ueno, K.; Oshikiri, T.; Sun, Q.; Shi, X.; Misawa, H. Solid-State Plasmonic Solar Cells. Chem. Rev. 2018, 118 (6), 2955-2993. https://doi.org/10.1021/acs.chemrev.7b00235.

(9) Green, M. A.; Pillai, S. Harnessing Plasmonics for Solar Cells. Nat. Photonics 2012, 6 (3), 130-132. https://doi.org/10.1038/nphoton.2012.30.

(10) Chang, W.-S.; Lassiter, J. B.; Swanglap, P.; Sobhani, H.; Khatua, S.; Nordlander, P.; Halas, N. J.; Link, S. A Plasmonic Fano Switch. Nano Lett. 2012, 12 (9), 4977-4982. https://doi.org/10.1021/nl302610v.

(11) Duan, X.; Kamin, S.; Liu, N. Dynamic Plasmonic Colour Display. Nat. Commun. 2017, 8, 14606. https://doi.org/10.1038/ncomms14606.

(12) Zeng, S.; Sreekanth, K. V.; Shang, J.; Yu, T.; Chen, C.-K.; Yin, F.; Baillargeat, D.; Coquet, P.; Ho, H.-P.; Kabashin, A. V.; Yong, K.-T. Graphene-Gold Metasurface Architectures for 
Ultrasensitive Plasmonic Biosensing. Adv. Mater. 2015, 27 (40), 6163-6169. https://doi.org/10.1002/adma.201501754.

(13) Engheta, N.; Salandrino, A.; Alù, A. Circuit Elements at Optical Frequencies: Nanoinductors, Nanocapacitors, and Nanoresistors. Phys. Rev. Lett. 2005, 95 (9), 095504. https://doi.org/10.1103/PhysRevLett.95.095504.

(14) Davis, T. J.; Gomez, D. E.; Roberts, A. Plasmonic Circuits for Manipulating Optical Information. Nanophotonics 2017, 6 (3), 543-559. https://doi.org/10.1515/nanoph-2016$\underline{0131 .}$.

(15) Engheta, N. Circuits with Light at Nanoscales: Optical Nanocircuits Inspired by Metamaterials. $\quad$ Science $2007, \quad 317 \quad$ (5845), $1698-1702$. https://doi.org/10.1126/science.1133268.

(16) Abbasi, F.; Engheta, N. Roles of Epsilon-near-Zero (ENZ) and Mu-near-Zero (MNZ) Materials in Optical Metatronic Circuit Networks. Opt. Express, 2014, 22 (21), 25109 25119. https://doi.org/10.1364/OE.22.025109.

(17) Liu, R.; Roberts, C. M.; Zhong, Y.; Podolskiy, V. A.; Wasserman, D. Epsilon-Near-Zero Photonics Wires. ACS Photonics 2016, 3 (6), 1045-1052. https://doi.org/10.1021/acsphotonics.6b00120.

(18) Xu, Y.; Tucker, E.; Boreman, G.; Raschke, M. B.; Lail, B. A. Optical Nanoantenna Input Impedance. ACS Photonics 2016, 3 (5), 881-885. https://doi.org/10.1021/acsphotonics.6b00128.

(19) Gosciniak, J.; Nielsen, M. G.; Markey, L.; Dereux, A.; Bozhevolnyi, S. I. Power Monitoring in Dielectric-Loaded Plasmonic Waveguides with Internal Wheatstone Bridges. Opt. Express, 2013, 21 (5), 5300-5308. https://doi.org/10.1364/OE.21.005300.

(20) Li, Y.; Liberal, I.; Engheta, N. Metatronic Analogues of the Wheatstone Bridge. J. Opt. Soc. Am. B, 2016, 33 (2), A72-A79. https://doi.org/10.1364/JOSAB.33.000A72.

(21) Alù, A.; Engheta, N. Enabling a New Degree of Wave Control with Metamaterials: A Personal Perspective. J. Opt. 2017, 19 (8), 084008. https://doi.org/10.1088/20408986/aa7790.

(22) Li, Y.; Engheta, N. Capacitor-Inspired Metamaterial Inductors. Phys. Rev. Appl. 2018, 10 (5), 054021. https://doi.org/10.1103/PhysRevApplied.10.054021.

(23) Sun, Y.; Edwards, B.; Alù, A.; Engheta, N. Experimental Realization of Optical Lumped Nanocircuits at Infrared Wavelengths. Nat. Mater. 2012, 11 (3), 208-212. https://doi.org/10.1038/nmat3230.

(24) Caglayan, H.; Hong, S.-H.; Edwards, B.; Kagan, C. R.; Engheta, N. Near-Infrared Metatronic Nanocircuits by Design. Phys. Rev. Lett. 2013, 111 (7), 073904. https://doi.org/10.1103/PhysRevLett.111.073904.

(25) Li, Y.; Liberal, I.; Giovampaola, C. D.; Engheta, N. Waveguide Metatronics: Lumped Circuitry Based on Structural Dispersion. Sci. Adv. 2016, 2 (6), e1501790. https://doi.org/10.1126/sciadv.1501790.

(26) Li, Y.; Zhang, Z. Experimental Verification of Guided-Wave Lumped Circuits Using Waveguide Metamaterials. Phys. Rev. Appl. 2018, 9 (4), 044024. https://doi.org/10.1103/PhysRevApplied.9.044024. 
(27) Hughes, T. W.; Fan, S. Plasmonic Circuit Theory for Multiresonant Light Funneling to a Single Spatial Hot Spot. Nano Lett. 2016, $16 \quad$ (9), 5764-5769. https://doi.org/10.1021/acs.nanolett.6b02474.

(28) Zhu, J.; Zhang, L.; Jiang, S.; Ou, J.-Y.; Liu, Q. H. Selective Light Trapping of Plasmonic Stack Metamaterials by Circuit Design. Nanoscale 2020, 12 (3), 2057-2062. https://doi.org/10.1039/C9NR07937H.

(29) Liu, N.; Wen, F.; Zhao, Y.; Wang, Y.; Nordlander, P.; Halas, N. J.; Alù, A. Individual Nanoantennas Loaded with Three-Dimensional Optical Nanocircuits. Nano Lett. 2013, 13 (1), 142-147. https://doi.org/10.1021/n1303689c.

(30) Shi, J.; Monticone, F.; Elias, S.; Wu, Y.; Ratchford, D.; Li, X.; Alù, A. Modular Assembly of Optical Nanocircuits. Nat. Commun. 2014, $5 \quad$ (1), 3896. https://doi.org/10.1038/ncomms4896.

(31) Park, K. J.; Huh, J.-H.; Jung, D.-W.; Park, J.-S.; Choi, G. H.; Lee, G.; Yoo, P. J.; Park, H.G.; Yi, G.-R.; Lee, S. Assembly of “3D” Plasmonic Clusters by "2D” AFM Nanomanipulation of Highly Uniform and Smooth Gold Nanospheres. Sci. Rep. 2017, 7 (1), 1-10. https://doi.org/10.1038/s41598-017-06456-w.

(32) Abasahl, B.; Santschi, C.; Martin, O. J. F. Quantitative Extraction of Equivalent Lumped Circuit Elements for Complex Plasmonic Nanostructures. ACS Photonics 2014, 1 (5), 403 407. https://doi.org/10.1021/ph400101w.

(33) Eftekhari, F.; Gómez, D. E.; Davis, T. J. Measuring Subwavelength Phase Differences with a Plasmonic Circuit - an Example of Nanoscale Optical Signal Processing. Opt. Lett. 2014, 39 (10), 2994. https://doi.org/10.1364/OL.39.002994.

(34) Benz, F.; Nijs, B. de; Tserkezis, C.; Chikkaraddy, R.; Sigle, D. O.; Pukenas, L.; Evans, S. D.; Aizpurua, J.; Baumberg, J. J. Generalized Circuit Model for Coupled Plasmonic Systems. Opt. Express, 2015, 23 (26), 33255-33269. https://doi.org/10.1364/OE.23.033255.

(35) Davis, T. J.; Gómez, D. E. Colloquium: An Algebraic Model of Localized Surface Plasmons and Their Interactions. Rev. Mod. Phys. 2017, 89 (1), 011003. https://doi.org/10.1103/RevModPhys.89.011003.

(36) Attaran, A.; Emami, S. D.; Soltanian, M. R. K.; Penny, R.; behbahani, F.; Harun, S. W.; Ahmad, H.; Abdul-Rashid, H. A.; Moghavvemi, M. Circuit Model of Fano Resonance on Tetramers, Pentamers, and Broken Symmetry Pentamers. Plasmonics 2014, 9 (6), 13031313. https://doi.org/10.1007/s11468-014-9743-y.

(37) Ma, P.; Gao, L.; Ginzburg, P.; Noskov, R. E. Nonlinear Nanophotonic Circuitry: Tristable and Astable Multivibrators and Chaos Generator. Laser Photonics Rev. 2020, 14 (3), 1900304. https://doi.org/10.1002/lpor.201900304.

(38) Alù, A.; Engheta, N. All Optical Metamaterial Circuit Board at the Nanoscale. Phys. Rev. Lett. 2009, 103 (14), 143902. https://doi.org/10.1103/PhysRevLett.103.143902.

(39) Lassiter, J. B.; Sobhani, H.; Fan, J. A.; Kundu, J.; Capasso, F.; Nordlander, P.; Halas, N. J. Fano Resonances in Plasmonic Nanoclusters: Geometrical and Chemical Tunability. Nano Lett. 2010, 10 (8), 3184-3189. https://doi.org/10.1021/nl102108u.

(40) Hentschel, M.; Dregely, D.; Vogelgesang, R.; Giessen, H.; Liu, N. Plasmonic Oligomers: The Role of Individual Particles in Collective Behavior. ACS Nano 2011, 5 (3), 2042-2050. https://doi.org/10.1021/nn103172t. 
(41) Coenen, T.; Schoen, D. T.; Mann, S. A.; Rodriguez, S. R. K.; Brenny, B. J. M.; Polman, A.; Brongersma, M. L. Nanoscale Spatial Coherent Control over the Modal Excitation of a Coupled Plasmonic Resonator System. Nano Lett. 2015, 15 (11), 7666-7670. https://doi.org/10.1021/acs.nanolett.5b03614.

(42) Von Cube, F.; Irsen, S.; Diehl, R.; Niegemann, J.; Busch, K.; Linden, S. From Isolated Metaatoms to Photonic Metamaterials: Evolution of the Plasmonic Near-Field. Nano Lett. 2013, 13 (2), 703-708. https://doi.org/10.1021/nl3043757.

(43) Lin, J.; Qiu, M.; Zhang, X.; Guo, H.; Cai, Q.; Xiao, S.; He, Q.; Zhou, L. Tailoring the Lineshapes of Coupled Plasmonic Systems Based on a Theory Derived from First Principles. Light Sci. Appl. 2020, 9 (1), 158. https://doi.org/10.1038/s41377-020-00386-5.

(44) Meng, Y.; Zhang, Q.; Lei, D.; Li, Y.; Li, S.; Liu, Z.; Xie, W.; Leung, C. W. PlasmonInduced Optical Magnetism in an Ultrathin Metal Nanosphere-Based Dimer-on-Film Nanocavity. Laser Photonics Rev. 2020, $14 \quad$ (9), 2000068. https://doi.org/10.1002/lpor.202000068.

(45) Rahmani, M.; Lei, D. Y.; Giannini, V.; Lukiyanchuk, B.; Ranjbar, M.; Liew, T. Y. F.; Hong, M.; Maier, S. A. Subgroup Decomposition of Plasmonic Resonances in Hybrid Oligomers: Modeling the Resonance Lineshape. Nano Lett. 2012, 12 (4), 2101-2106. https://doi.org/10.1021/nl3003683.

(46) Johnson, P. B.; Christy, R. W. Optical Constants of the Noble Metals. Phys. Rev. B 1972, 6, 4370-4379. https://doi.org/10.1103/PhysRevB.6.4370. 